\title{
Tracing the glycogen cells with Protocadherin 12 during mouse placenta development
}

Stéphanie Bouillot, Christine Rampon, Emmanuelle Tillet and Philippe Huber ${ }^{1}$

INSERM; CEA; Université J. Fourier EMI 02-19, Laboratoire de Développement et Vieillissement de l'Endothélium, Grenoble, France

Running title: Protocadherin 12-positive cells in the mouse placenta

${ }^{1}$ Corresponding Author: CEA-Grenoble, DRDC-DVE, 17 rue des Martyrs, 38054 Grenoble cedex 9, France. Tel.: +33-438-78-41-18; fax: +33-438-78-49-64. E-mail address: phuber@cea.fr. 


\section{ABSTRACT}

Among the different trophoblast subtypes of the mouse placenta, the glycogen cells (GC) are one of the trophoblast subtypes that invade the decidua. We previously established that GC specifically expressed protocadherin 12 (PCDH12). In this paper, we investigated the origin of the PCDH12-positive cells and we characterized their fate in the maternal tissues. Our data indicate that they directly originate from the central part of the ectoplacental cone at embryonic day (E) 7.5. PCDH12-positive cells start to accumulate glycogen from E10.5 and the first migrating cells could be observed from this age. Unlike other placental and decidual cells, GC do not express P-cadherin, which may explain their migration properties in this organ. In the decidua, GC settle in the vicinity of the maternal vascular sinuses but do not incorporate in the endothelium. By the end of gestation (E17.5), most GC islets of the decidua enter into a lytic phase and form large lacunae. These lacunae, filled with glycogen, may provide a substantial source of energy at the end of gestation or during delivery. Our data suggest that spongiotrophoblasts and GC are two independent lineages and we bring insights into GC migration and fate. 


\section{INTRODUCTION}

The mouse placenta is composed from embryonic day (E) 10.5 of four layers namely, the chorionic plate on the embryonic side, the labyrinth, the spongiotrophoblast layer or junctional zone and the trophoblast giant cell layer lining the maternal decidua [1-3]. The structure and properties of the labyrinth in the feto-maternal exchanges as well as the role of the giant cells in implantation have been extensively studied. In contrast, the precise function of the spongiotrophoblast layer is unknown although its integrity is required for fetus viability $[4,5]$. It could act as structural support for the developing villous structure of the labyrinth and is also known to express several unique factors, including hormones [6, 7]. Unlike the labyrinth, the spongiotrophoblast layer does not contain capillaries; it is however traversed by the central maternal artery and the lateral maternal veins thereby allowing the circulation of maternal blood in the labyrinth and the subsequent molecular exchanges with the fetal circulatory system in this layer [8].

Two types of cytotrophoblasts are located in the spongiotrophoblast layer: the spongiotrophoblasts and from E12.5, the "glycogen cells" (GC) [8, 9]. The spongiotrophoblasts are basophilic cells directly originating from the ectoplacental cone. The evidence of this is based on histological studies and expression patterns of genes that in the placenta are restricted to the ectoplacental cone and later in the spongiotrophoblast layer (e.g. tpbpa and Flt1) [10, 11]. GC are characterized by a vacuolated glycogen-rich cytoplasm and appear as compact cell islets. Because they emerge in this zone after placenta layer organization and since both cell types express the Tpbpa gene, it was suggested that GC could represent a specialized subtype of spongiotrophoblasts [1-3]. GC thereafter migrate in the decidua, beyond the giant cells. Therefore, it has been suggested that the mouse GC could be analogous to the human extravillous cytotrophoblasts responsible for the invasion of the 
decidua and myometrium [1]. A second similarity resides in the fact that the distal extravillous cytotrophoblasts in humans also contain high amounts of glycogen. As opposed to human extravillous cytotrophoblasts, little data is available on GC.

We recently showed that protocadherin 12 (PCDH12) was highly and specifically expressed by GC at E12.5 [12]. PCDH12 is a member of the cadherin superfamily that was originally identified in endothelial cells [13]. Two goals prompted the present work. One was to define the origin of the PCDH12-positive cells, which potentially represent GC precursors, before placenta organization (before E10.5). The other was to determine the fate of GC after decidual invasion. PCDH12 immunolabeling proved to be a better marker to identify GC than the histological detection of glycogen. 


\section{MATERIALS AND METHODS}

\section{Animals}

Mice were treated in accord with the European guidelines of animal care. Female CD1 mice (outbred strain, Charles River) were bred overnight with male CD1 mice and the presence of the vaginal plug on the morning following mating was considered day 0.5 of gestation.

\section{Antibodies}

The rabbit antibody anti-mouse PCDH12 was previously described [12] and we used it at a concentration of $5 \mu \mathrm{g} / \mathrm{mL}$ for immunohistology and at $0.1 \mu \mathrm{g} / \mathrm{mL}$ for Western blot analysis. Rat anti-mouse CD31 (MEC13) was a gift from Dr A. Vecchi [14] and was used as undiluted hybridoma supernatant. The anti-P-cadherin antibody from Zymed Laboratories was used at 5 $\mu \mathrm{g} / \mathrm{mL}$. As secondary antibodies, the cyanine 3-conjugated antibodies were from Jackson Immunoresearch Laboratories and the alexa 488-conjugated antibodies were from Molecular Probes; all were used at recommended dilutions. The peroxidase-conjugated anti-rabbit antibody used in Western blot was from Biorad and used at 1:3,000 dilution.

\section{Histology}

At indicated ages, pregnant females were killed by cervical dislocation and conceptuses were dissected in PBS. For immunolocalization, tissues were snap-frozen in OCT compound and sectioned at $10-\mu \mathrm{m}$ with a cryomicrotome (Leica). Sections were permeabilized in paraformaldehyde $4 \%$, Triton $0.5 \%$-PBS for $5 \mathrm{~min}$, fixed in $4 \%$ paraformaldehyde-PBS for 20 min, saturated with $2 \% \mathrm{BSA} / \mathrm{PBS}$ and incubated with primary and secondary antibodies, all at room temperature, using standard procedures. Sections were incubated for 2 min in Hoechst 
33258 (Sigma) for nuclear staining. Fluorescent slides were mounted in Fluorsave reagent (Calbiochem). For hematoxylin/eosin or periodic acid-Schiff (all from Sigma) staining, paraffin sections $(5 \mu \mathrm{m})$ were prepared using standard procedures, from paraformaldehydeprefixed tissues. Slides were mounted in Entellan or Aquatex (Merck). Slides were observed under a fluorescent microscope (Axioplan, Zeiss) and pictures were made with a digital camera (Spot 2, Diagnostic Instruments). For semi-thin sections, tissue samples were fixed 2 $\mathrm{h}$ in $2.5 \%$ glutaraldehyde/0.1 M sodium cacodylate $\mathrm{pH} 7.2$ and then postfixed $45 \mathrm{~min}$ in $1 \%$ osmium tetroxid in $0.15 \mathrm{M}$ sodium cacodylate. Following ethanol dehydration, samples were incubated $30 \mathrm{~min}$ in propylene oxide and embedded in epoxy resin (Epon Embed 812, Electron Microscopy Sciences). Semithin sections $(1 \mu \mathrm{m})$ were obtained from an ultramicrotome (Ultracut, Leica), stained with toluidine blue and observed with above microscope.

\section{Immunoblot analysis}

Protein extracts were obtained by homogenization with a Polytron in a solution containing 20 $\mathrm{mM}$ Tris- $\mathrm{HCl} \mathrm{pH}$ 7.2, $1 \mathrm{mM}$ EDTA, $1 \mathrm{mM}$ EGTA, $1 \mathrm{mM} \mathrm{Na} \mathrm{VO}_{4}, 10 \mathrm{mM} \beta-$ glycerophosphate, $5 \mathrm{mM} \mathrm{NaF}, 1 \mathrm{mM}$ dithiotreitol, $0.27 \mathrm{M}$ sucrose, $4 \mu \mathrm{g} / \mathrm{mL}$ leupeptin, $1 \%$ Triton, $0.5 \%$ NP40 and the antiprotease cocktail from Roche. Protein concentration was determined with a Micro-BCA kit (Pierce), using BSA as standard. Proteins were electrophorezed in denaturing polyacrylamide (6\%) gels (SDS-PAGE) in reducing conditions, transferred onto Hybond ECL membrane (Amersham) and incubated with antibodies as described above. The molecular mass standard was from Biorad. 


\section{RESULTS}

\section{Ontogeny and migration of the PCDH12-positive cells during mouse placenta development}

PCDH12 was originally characterized in endothelial cells [13]. However, immunohistological detection of this protein in the decidua vascular compartment is restricted to its periphery at early gestation ages (until E7.5) [12]. In contrast, PCDH12 staining was conspicuous in GC of E12.5 placentas [12]. Therefore, we used PCDH12 immunolabeling to follow these cells during placenta development. Sections were co-immunolabeled for CD31, a specific marker of endothelial cells [14], which was useful to identify the placenta anatomical layers.

We first wondered whether PCDH12 could be an earlier GC marker than glycogen accumulation in the cytoplasm and whether PCDH12 immunostaining could be of interest to determine the origin of these cells. PCDH12 staining was not detected in blastocysts (not shown) or in E6.5 embryos (Fig. 1A). The onset of embryonic PCDH12 expression was at E7.5 in the center of the ectoplacental cone (Fig. 1A). At this embryonic age, the cells did not form such tight clusters as previously shown by confocal microscopy at E12.5 [12]. Interestingly in some embryos, a few PCDH12-positive cells of unknown origin were also located outside of the embryo, along the uterine crypt, as illustrated in Fig 1A (arrow). However besides this location, no PCDH12-positive cells could ever be observed in the decidua before E10.5. From E9.5, PCDH12-positive cells formed a collection of aggregates, located at the periphery of the ectoplacental cone (E9.5) or in the spongiotrophoblast layer (E10.5 onwards), harboring a strong PCDH12 immunolabeling at the cell-cell junctions (Fig 1A).

We next examined GC migration after placenta layer organization. Starting at E10.5, but most prominent from E12.5, PCDH12-positive cells were detected in the decidua (i.e., beyond the giant cell layer). At E12.5, the PCDH12-positive cells in the decidua were either assembled in 
islets or in cords (Fig. 1A). Occasionally, PCDH12-positive cells, intermingled with spongiotrophoblasts, were observed in the labyrinthine layer, suggesting projections or "pegs" of the spongiotrophoblast layer (not shown). At E17.5, most PCDH12-positive cells were localized in the decidua but never in the myometrium (Fig. 1A). Altogether, these data indicate that PCDH12-positive cells first appear at E7.5 and migrate from E10.5 into the decidua interstitium where they eventually represent a substantial part of total cell number in this layer.

GC, first identified by their glycogen content, could be detected from E12.5 in the spongiotrophoblast layer [8,9]. We attempted to re-examine the question of glycogen apparition in these cells at earlier stages. Glycogen was detected using the periodic acidSchiff (PAS) reaction. Rare PAS-positive cells were detected at E6.5, E7.5 (arrows in Fig. 1B) or E9.5 (not shown). In contrast, numerous PAS-positive cells, assembled in islets, were consistently observed at E10.5 (arrow in Fig. 1B). However, PAS labeling was weaker than later on (not shown), suggesting that glycogen cell content was not maximal at E10.5. We previously established that PCDH12 expression in E12.5 trophoblast cells was specific to GC [12]. PCDH12 expression may thus be an earlier marker for these cells than glycogen storage. To precisely observe migrating GC, we prepared semi-thin sections from E12.5 placentas stained with toluidine blue. Under these conditions, GC appeared highly vacuolated because of glycogen loss during tissue preparation (arrows in Fig. 1C, left). Within the GC islets, cells were tightly apposed one to another, suggesting direct cell-cell contacts. GC islets adopted, on their way to the decidual layer, a typical migration front (Fig. 1C, right panel), suggesting that GC migrate collectively and not individually.

\section{Glycogen cell fate in the decidua}


In the decidua, the maternal uterine natural killer cells also harbor high glycogen content $[8$, 9]. This common feature with GC complicated the view of GC fate in this layer. However, the uterine natural killer cells do not express PCDH12. This is based on the fact that before GC migration, no PCDH12-positive cells could be detected in the decidua although numerous glycogen-containing cells were present in this layer (Fig. 1B, arrowhead). Thus, we used PCDH12 immunolabeling to examine GC fate at late gestation (E17.5). Sections were coimmunolabeled for CD31 to identify the vascular sinuses of the decidua. GC migration in the decidua often led to a close association with the vascular sinuses. A direct interaction between PCDH12-positive and endothelial cells was frequently observed, as illustrated in Fig. 2A, left panel (arrow). However, PCDH12-positive cells were never incorporated in the endothelium. We also consistently observed lacunae that were lined by PCDH12-positive cells (Fig. 2A, right). These lacunae were devoid of cells as shown by Hoechst staining and were located in the vicinity of these vascular sinuses. To further observe these lacunae, we stained E17.5 placenta sections with PAS and hematoxylin (Fig. 2B). The vascular sinuses could be identified by their blood cell content (arrowhead) and the GC lacunae (indicated by an *) by the PAS-positive cells located at their periphery. At this stage, GC showed extensive lysis (arrow) that could be distinguished from the vacuolated pre-lytic cells by the absence of a continuous plasma membrane. This feature suggests that these lacunae could be formed by GC liquefaction. Interestingly, no blood cell could ever be detected in these lacunae, indicating that the vascular system did not communicate with them. In addition, no fibrous material could be identified in these lacunae by hematoxylin-eosin-saffron staining of E17.5 placenta sections (not shown). Altogether, these observations suggest that, at the end of gestation, GC could liberate their cytosolic material, including glycogen, in vascular sinus proximity. 


\section{P-cadherin and PCDH12 are exclusive markers of placental cells}

P-cadherin was previously shown to be extensively expressed in trophoblasts at E7 and E14 as well as in decidual cells [15]. It is well known that cadherin expression controls cell detachment and migration [16]. We wondered whether PCDH12-positive cells express Pcadherin, as same cadherin family member expression could impede their migratory pathway in the placenta. At E6.5, P-cadherin was only expressed by decidual cells (Fig. 3). Later on, Pcadherin labeling was conspicuous on both maternal and embryonic sides of the placenta. However, PCDH12-positive cells were not labeled by P-cadherin antibodies (Fig. 3). Hence, these cells have a different homotypic adhesive code than other placental and decidual cells, in accordance with their behavior in this organ.

\section{Alteration of PCDH12 molecular mass in late placenta development}

We previously reported that PCDH12 apparent molecular mass was higher in the placenta (E12.5) compared to other organs [12]. This electrophoretic mobility shift was abolished after treatment with $\mathrm{N}$-glycosidase suggesting that the difference was due to the carbohydrate moiety. Here, we compared the electrophoretic migration of PCDH12 from placenta extracts at three different gestation ages (Fig. 4). We noticed that PCDH12 electrophoretic mobility gradually shifted from the high to the low molecular masses between E12.5 and E19, suggesting that PCDH12 eventually maturated in the placenta like in other tissues [12]. 


\section{DISCUSSION}

In this paper, we show that PCDH12-positive cells originate from the ectoplacental cone where they rapidly form tight islets. As GC express high amounts of PCDH12 and form similar islets, it is possible that the PCDH12-positive cells of the E7.5 ectoplacental cone represent GC progenitors. If this is correct, it refutes the hypothesis of a differentiation from the spongiotrophoblasts in the spongiotrophoblast layer at E12.5.

\section{Ectoplacental origin of the PCDH12-positive cells}

A large number of PCDH12-positive cells appears at E7.5, representing a substantial proportion of the ectoplacental cone cells, whereas none were visible at E6.5. This may indicate that they are, at this stage, rapidly proliferating cells. In agreement with this, the PCDH12-positive cells were localized in the core of the ectoplacental cone at E7.5, an area known to contain fast dividing cells [17]. Afterwards, the PCDH12-positive cells were located in the ectoplacental cone edge (Fig. 1A). Altogether, these data suggest that GC stem from the center of the ectoplacental cone and migrate at its periphery where they form characteristic islets.

The bHLH transcription factor Ascl2 (also called Mash2) is expressed in all parts of the ectoplacental cone at E7.5. However, expression of its obligate binding partners, Tcf12 (also called Alf1) and Itf2, is restricted to the inner part of the ectoplacental cone [4]. Thus, proliferation of PCDH12-positive cell precursors may be under control of Ascl2 transcriptional activity.

\section{Glycogen cell adhesion and migration}


As shown here, the PCDH12-positive cells do not express P-cadherin, in an environment of Pcadherin-expressing cells. Likewise, this feature may explain why GC assemble independently of the other cell types and easily migrate from the spongiotrophoblast layer to the decidua. Other protocadherins were shown to be required for cell sorting and histogenesis [18-20]. Whether PCDH12 itself is sufficient to promote such a tight adhesion between GC or whether other homophilic adhesive molecules are present at GC junctions is under investigation.

Alteration of PCDH12 molecular mass during placenta development probably results from carbohydrate modifications. It is interesting to note that there is a time correlation between GC migration and PCDH12 molecular mass shift. Alteration of cadherin glycosylation is known to change cell-cell adhesive activity. For example, the overglycosylation of E and Ncadherin has been correlated with increased cellular motility, invasiveness and metastasis [21, 22]. Thus, the different glycosylation patterns of PCDH12 may reflect a modification in its activity during and after invasion.

Using PAS staining, we could detect glycogen within GC from E10.5, earlier than previously described, indicating that glycogen accumulation is a process that occurs before GC migration in the decidua. As far as we know, PCDH12 is the first non-diffusible specific marker protein that can be used to identify GC in the mouse placenta.

From E10.5-12.5, GC collectively migrate towards the vascular sinuses of the decidua. Hence, GC are similar to the human extravillous cytotrophoblasts that invade the decidua interstitium. They do not however integrate or replace the maternal endothelium like those human extravillous cytotrophoblasts responsible of endovascular infiltration of the spiral arteries. Interestingly, trophoblast giant cells were recently shown to have the capacity to replace the maternal endothelium [23]. Once in the decidua, GC enter into a cytolytic process conducting to the release of their intracellular components, including glycogen and probably hormones, in 
Bouillot et al. PL-05-000066

the decidua interstitium. It is likely that this material is used as energy supply and for hormonal regulation of late gestation and/or fetus delivery.

\section{ACKNOWLEDGMENTS}

This work has been supported by the Ligue contre le Cancer and by the Fondation pour la Recherche Médicale. 


\section{REFERENCES}

[1] Georgiades P, Ferguson-Smith AC,Burton GJ. Comparative developmental anatomy of the murine and human definitive placentae. Placenta 2002;23:3-19.

[2] Cross JC, Baczyk D, Dobric N, Hemberger M, Hughes M, Simmons DG, Yamamoto H,Kingdom JC. Genes, development and evolution of the placenta. Placenta $2003 ; 24: 123-30$.

[3] Simmons DG,Cross JC. Determinants of trophoblast lineage and cell subtype specification in the mouse placenta. Dev Biol 2005.

[4] Guillemot F, Nagy A, Auerbach A, Rossant J,Joyner AL. Essential role of Mash-2 in extraembryonic development. Nature 1994;371:333-6.

[5] Tanaka M, Gertsenstein M, Rossant J,Nagy A. Mash2 acts cell autonomously in mouse spongiotrophoblast development. Dev Biol 1997;190:55-65.

[6] Iwatsuki K, Shinozaki M, Sun W, Yagi S, Tanaka S,Shiota K. A novel secretory protein produced by rat spongiotrophoblast. Biol Reprod 2000;62:1352-9.

[7] Cross JC, Hemberger M, Lu Y, Nozaki T, Whiteley K, Masutani M,Adamson SL. Trophoblast functions, angiogenesis and remodeling of the maternal vasculature in the placenta. Mol Cell Endocrinol 2002;187:207-12.

[8] Adamson SL, Lu Y, Whiteley KJ, Holmyard D, Hemberger M, Pfarrer C,Cross JC. Interactions between trophoblast cells and the maternal and fetal circulation in the mouse placenta. Dev Biol 2002;250:358-73.

[9] Redline RW,Lu CY. Localization of fetal major histocompatibility complex antigens and maternal leukocytes in murine placenta. Implications for maternal-fetal immunological relationship. Lab Invest 1989;61:27-36. 
[10] Lescisin KR, Varmuza S,Rossant J. Isolation and characterization of a novel trophoblast-specific cDNA in the mouse. Genes Dev 1988;2:1639-46.

[11] He Y, Smith SK, Day KA, Clark DE, Licence DR,Charnock-Jones DS. Alternative splicing of vascular endothelial growth factor (VEGF)-R1 (FLT-1) pre-mRNA is important for the regulation of VEGF activity. Mol Endocrinol 1999;13:537-45.

[12] Rampon C, Prandini MH, Bouillot S, Pointu H, Tillet E, Frank R, Vernet M,Huber P. Protocadherin 12 (VE-cadherin 2) is expressed in endothelial, trophoblast, and mesangial cells. Exp Cell Res 2005;302:48-60.

[13] Telo P, Breviario F, Huber P, Panzeri C,Dejana E. Identification of a novel cadherin (vascular endothelial cadherin-2) located at intercellular junctions in endothelial cells. J Biol Chem 1998;273:17565-72.

[14] Vecchi A, Garlanda C, Lampugnani MG, Resnati M, Matteucci C, Stoppacciaro A, Schnurch H, Risau W, Ruco L, Mantovani A,Dejana E. Monoclonal antibodies specific for endothelial cells of mouse blood vessels. Their application in the identification of adult and embryonic endothelium. Eur J Cell Biol 1994;63:247-54.

[15] Nose A,Takeichi M. A novel cadherin cell adhesion molecule: its expression patterns associated with implantation and organogenesis of mouse embryos. J Cell Biol 1986;103:2649-58.

[16] Perez-Moreno M, Jamora C,Fuchs E. Sticky business: orchestrating cellular signals at adherens junctions. Cell 2003;112:535-48.

[17] Mehrotra PK. Ultrastructure of mouse ectoplacental cone cells. Biol Struct Morphog 1988;1:63-8.

[18] Bradley RS, Espeseth A,Kintner C. NF-protocadherin, a novel member of the cadherin superfamily, is required for Xenopus ectodermal differentiation. Curr Biol 1998;8:325-34. 
[19] Kim SH, Jen WC, De Robertis EM,Kintner C. The protocadherin PAPC establishes segmental boundaries during somitogenesis in xenopus embryos. Curr Biol 2000;10:821-30.

[20] Kuroda H, Inui M, Sugimoto K, Hayata T,Asashima M. Axial protocadherin is a mediator of prenotochord cell sorting in Xenopus. Dev Biol 2002;244:267-77.

[21] Guo HB, Lee I, Kamar M,Pierce M. N-acetylglucosaminyltransferase V expression levels regulate cadherin-associated homotypic cell-cell adhesion and intracellular signaling pathways. J Biol Chem 2003;278:52412-24.

[22] Geng F, Shi BZ, Yuan YF,Wu XZ. The expression of core fucosylated E-cadherin in cancer cells and lung cancer patients: prognostic implications. Cell Res 2004;14:42333.

[23] Hemberger M, Nozaki T, Masutani M,Cross JC. Differential expression of angiogenic and vasodilatory factors by invasive trophoblast giant cells depending on depth of invasion. Dev Dyn 2003;227:185-91. 


\section{FIGURE LEGENDS}

\section{Figure 1: Origin and migration of glycogen cells during placenta development}

A. Decidua and placenta cryosections $(10 \mu \mathrm{m})$ were immunolabeled with anti-PCDH12 antibodies (red). The anti-CD31 antibodies (green) were used to detect endothelial cells. The embryonic ages are indicated at the top left corner. The arrow shows the migration of PCDH12-positive cells along the uterine crypt. B. Detection of glycogen with PAS reagent (purple). Nuclei were counterstained with hematoxylin (blue). At E6.5 and E7.5, the arrows show glycogen-containing cells. At E10.5, the arrow shows a GC islet and the arrowhead, a uterine natural killer cell. The insert represents a GC islet at higher magnification in the spongiotrophoblast layer. C. Toluidine blue staining (left) and schematic representation (right) of a E12.5 semithin section $(1 \mu \mathrm{m})$ showing a GC islet migrating in the decidua. Abbreviations: e, embryo; ec, ectoplacental cone; c, ectoplacental cavity; d, decidua; 1, labyrinth layer; s, spongiotrophoblast layer. The white and black lines were added to indicate the limits between layers.

\section{Figure 2: Glycogen cell fate in the decidua}

A. PCDH12 (red) and CD31 (green) labeling of E17.5 decidua. Nuclei are counterstained in blue with Hoechst. The arrow shows, on the left panel, the interaction between endothelial cells and GC. The $\left(^{*}\right)$ on the right panel indicates a cell-devoid lacuna lined by GC. B. PAS/hematoxylin staining of a E17.5 decidua section showing a PCDH12 lacuna (*) with lysed GC (arrow), adjacent to a vascular vessel containing blood cells (arrowhead).

\section{Figure 3: PCDH12 and P-cadherin tissue distribution in the developing placenta}


P-cadherin (green) and PCDH12 (red) co-immunolabeling was performed on decidua/placenta sections at different embryonic ages, as indicated at the top left corner. For E6.5 sections, nuclei were stained with Hoechst (blue). Abbreviations are as in Fig. 1.

\section{Figure 4: Immunoblot analysis of PCDH12 in placenta extracts}

Protein extracts $(50 \mu \mathrm{g})$ were prepared from placentas at E12.5, E17.5 and E19, analyzed by SDS-PAGE $(6 \%)$ and immunoblotted with anti-PCDH12 antibodies. Dashed lines and arrowheads were added to indicate the shift in PCDH12 molecular mass from mid to late gestation. 